\title{
Microbiota fúngica da conjuntiva, da cana-de-açúcar e de anemófilos da região canavieira de Monte Belo - Minas Gerais
}

\author{
Fungic microbiota of normal conjunctiva, sugar-cane and \\ anemophilous fungi of the region of Monte Belo-Minas Gerais
}

\author{
Joyce Treinta Dalfré ${ }^{1}$ \\ João Paulo Brandão Rodrigiues ${ }^{2}$ \\ Bruno Guimarães Donato ${ }^{3}$ \\ Armando Giancoli Neto ${ }^{4}$ \\ Juliano Lopes de Carvalho ${ }^{5}$ \\ Daniel Iscold de Andrade Oliveira ${ }^{6}$ \\ Maria Aparecida Pereira ${ }^{7}$ \\ João Evangelista Fiorinis
}

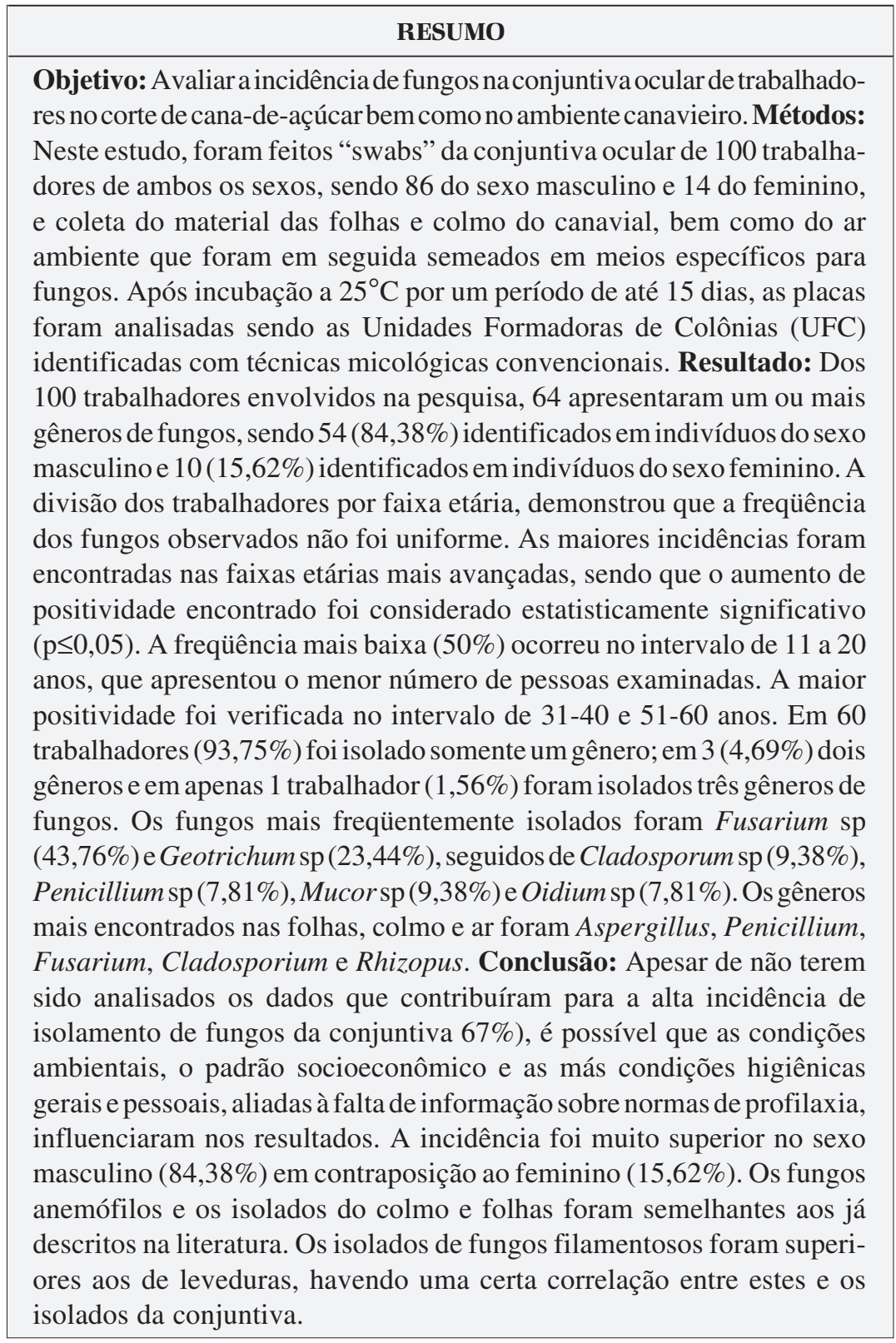

Descritores: Fungos/isolamento \& purificação; Conjuntiva/microbiologia; Oftalmopatias; Saccharum; Doenças dos trabalhadores agrícolas
Trabalho realizado na Universidade José do Rosário Vellano - UNIFENAS.

Acadêmica de Medicina da Universidade José do Rosário Vellano - UNIFENAS - Alfenas (MG) - Brasil.

2 Acadêmico de Medicina da UNIFENAS - Alfenas (MG) - Brasil.

${ }^{3}$ Acadêmico de Medicina da UNIFENAS - Alfenas (MG) - Brasil.

Acadêmico de Medicina da UNIFENAS - Alfenas (MG) - Brasil.

Médico Oftalmologista e Coordenador do Instituto de Olhos da UNIFENAS - Alfenas (MG) - Brasil.

${ }^{6}$ Mestre em Ciência Animal. Laboratório de Biologia e Fisiologia de Microrganismos - UNIFENAS - Alfenas (MG) - Brasil.

Acadêmica de Farmácia - UNIFENAS - Alfenas (MG) Brasil.

${ }^{8}$ Doutor em Microbiologia. Pesquisador Sênior. Laboratório de Biologia e Fisiologia de Microrganismos UNIFENAS - Alfenas (MG) - Brasil.

Endereço para correspondência: João Evangelista Fiorini - Laboratório de Biologia e Fisiologia de Microrganimos - UNIFENAS - Rodovia MG 179-Km 0-Alfenas - MG CEP 37130-000

E-mail: microrganismo@unifenas.br

Recebido para publicação em 04.01.2006

Última versão recebida em 13.12.2006

Aprovação em 13.03.2007 


\section{INTRODUÇÃO}

O olho, em constante contato com o meio ambiente, está exposto a diversos agentes patogênicos. Os fungos podem comprometer a maioria das estruturas oculares como pálpebra, conjuntiva, córnea e aparelho lacrimal ${ }^{(1)}$. A infecção ocular mais comum causada por fungos oportunistas é a ceratite micótica ${ }^{(2)}$. A presença de contaminação micótica assintomática da conjuntiva e margem palpebral encontra-se na dependência principalmente da idade e de fatores ambientais ${ }^{(3)}$.

A epidemiologia da úlcera corneana por fungo filamentoso é diferente daquela causada por levedura. Úlceras corneanas causadas por fungos filamentosos geralmente ocorrem após traumatismos com material vegetal, pó de madeira ou corpos estranhos do solo, contaminados por terra, em olhos previamente sadios ${ }^{(4)}$. Ceratomicoses por fungos filamentosos constituem um problema ocupacional de trabalhadores no meio rural. Em contraste, as ceratomicoses causadas por leveduras, comumente, ocorrem em olhos com doenças corneanas pósexistentes $^{(5)}$.

O presente trabalho se propôs a avaliar a incidência de fungos filamentosos na conjuntiva ocular normal de trabalhadores no corte de cana-de-açúcar da região de Monte Belo - MG, bem como no ambiente canavieiro, folhas e colmo de vegetal.

\section{MÉTODOS}

\section{Coleta das amostras}

As amostras foram colhidas durante o mês de novembro de 2003 do olho esquerdo e direito de 100 trabalhadores dedicados ao corte de cana-de-açúcar durante suas atividades na Fazenda Monte Alegre, Monte Belo - MG.

Foram feitas duas coletas no próprio local - a primeira em 30 trabalhadores e a segunda em 70 trabalhadores. A temperatura variou entre $20-23^{\circ} \mathrm{C}$ com ventos moderados durante ambas as coletas.

Os trabalhadores não apresentavam patologias oculares e não faziam uso de quaisquer medicamentos.

\section{Procedimentos de coleta}

\section{Conjuntiva}

As amostras das conjuntivas foram colhidas com "swabs" estéreis umedecidos com solução salina estéril, fazendo-se a eversão palpebral, a fim de possibilitar a coleta do fundo do saco conjuntival inferior, tendo-se o cuidado de não tocar nas margens palpebrais. Em seguida, o material foi inoculado em tubos de ensaio contendo meio de transporte.

\section{Ar}

No segundo dia da coleta foram expostos ao ar 13 placas de Petri, contendo o meio de Sabouraud-glicose acidificado (SGA) em vários locais do canavial a uma altura de 1,20 metros do solo, em repouso, por um período de 30 minutos, em posição horizontal.

\section{Epiderme da cana-de-açúcar (folha e colmo)}

Foram retirados pequenos fragmentos das folhas e colmos da cana-de-açúcar e semeados na superfície do meio SGA, distribuído em placas de Petri. Tal coleta também foi realizada no segundo dia da pesquisa.

\section{Isolamento e identificação}

Todas as amostras colhidas foram semeadas na superfície do meio SGA, incubado a $25^{\circ} \mathrm{C}$ em câmara úmida, por um período de até 15 dias. Após o crescimento, foram realizados testes de identificação incluindo a análise de colônia gigante, aspecto macromorfológico e microscópico, envolvendo a realização de microcultivo em lâmina e análise bioquímica de fermentação e assimilação de fontes de carbono e nitrogênio e, para a confirmação dos gêneros de leveduras empregou-se galerias API - AUX (Bio Mèrieux).

\section{Método estatístico}

Foi utilizado o teste de Máxima Verossimilhança com fator de correção de Willians $(14,304)$ e nível de significância igual a $5 \%$, para investigação da incidência de positividade de fungos em conjuntiva normal, com o evoluir da idade.

\section{RESULTADOS}

Dos 100 trabalhadores envolvidos na pesquisa, 64 (64\%) apresentaram um ou mais gêneros de fungos, sendo $54(84,38 \%)$ identificados em indivíduos do sexo masculino e $10(15,62 \%)$ identificados em indivíduos do sexo feminino.

A divisão dos trabalhadores por faixa etária demonstrou que a frequiência de fungos observados por idade não foi uniforme. Este dado não pode ser considerado tendencioso uma vez que foram realizadas apenas duas coletas nos indivíduos presentes no momento da mesma (Tabela 1).

Pôde-se observar que as maiores incidências apresentam uma leve tendência para faixas etárias mais avançadas, sendo que o aumento da positividade encontrada nestas faixas etárias foi considerado estatisticamente significativo $(p \leq 0,05)$.

A freqüência mais baixa (50\%) ocorreu no intervalo de 11 a 20 anos, que apresentou o menor número de pessoas examinadas. A maior positividade foi verificada no intervalo de 31-40 e 51-60 anos (Tabela 1).

Em 60 trabalhadores foi isolado somente um gênero / indivíduo (93,37\%); em 3, dois gêneros e em apenas 1 trabalhador foi isolado três gêneros de fungos $(1,56 \%)$, alcançando um total de seis gêneros nos diversos trabalhadores (Tabela 3).

Os fungos mais frequientemente isolados foram Fusarium sp atingindo $43,76 \%$ de positividade e Geotrichum sp com 23,44\%, seguidos de Cladosporium sp (9,38\%), Penicillium sp (7,81\%), Mucor $\mathrm{sp}(6,25 \%)$ e Oidium $\mathrm{sp}(3,12 \%)$. Aspergillus $\mathrm{sp}$ e Rhizopus sp não foram isolados da conjuntiva (Tabela 2). Com 
relação à microbiota anemófila, de colmo e folhas, esta demonstrou semelhança com isolados conjuntival (Tabela 2).

Quanto ao número de culturas positivas, apresentando o crescimento de um único gênero ou em associação com outros, a freqüência entre os fungos isolados, bem como a freqüência entre o total de trabalhadores, os resultados se encontram sumarizados na tabela 3 .

\section{DISCUSSÃO}

O olho encontra-se em constante contato com o ambiente o que representa uma porta de entrada em potencial para microrganismos, os mais diversos, incluindo fungos, bactérias e vírus, patógenos ou não.

Em condições normais, os fungos de sacos conjuntivais, as vias lacrimais e os orifícios glandulares palpebrais, constituem o locais adequados para o alojamento e desenvolvimento de fungos, pois encontram condições uniformes de umidade, calor e proteção luminosa favoráveis ao saprofitismo. Aliada a estas condições existe ainda o fato de estarem a conjuntiva e anexos permanentemente em contato direto com o ar ambiente, no qual existe um teor de esporos, maior ou menor, na dependência de diversos fatores ${ }^{(6)}$. Em nosso trabalho es-

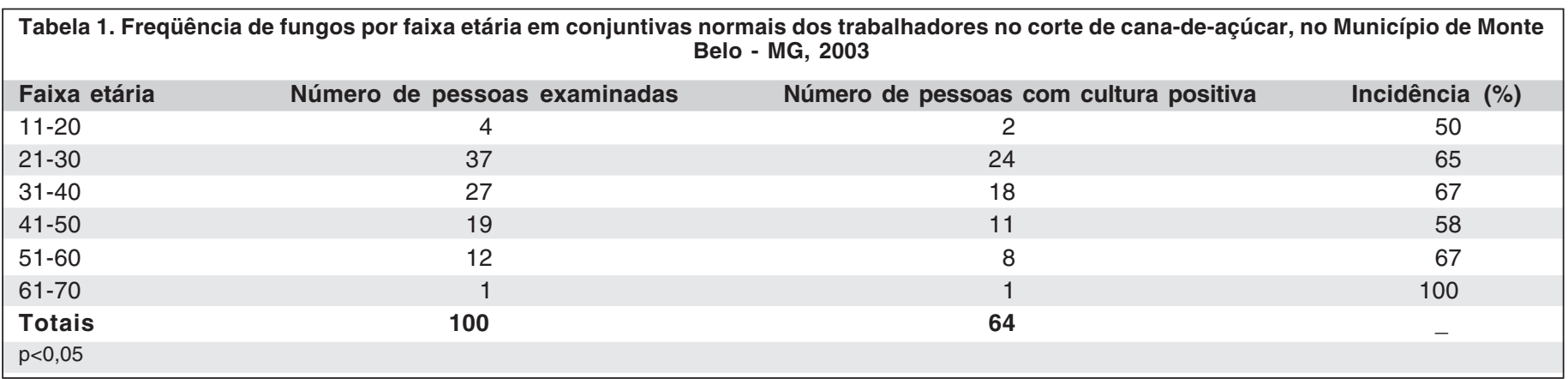

Tabela 2. Número de isolamentos e freqüência de fungos na conjuntiva normal de trabalhadores no corte de cana-de-açúcar, do ar e na superfície epidérmica do colmo e da folha da cana, no município de Monte Belo - MG, 2003

\begin{tabular}{|c|c|c|c|c|}
\hline Gênero dos fungos & $\begin{array}{c}\text { Conjuntiva } \\
\text { n (\%) }\end{array}$ & $\begin{array}{l}\text { Anemófila } \\
\text { n (\%) }\end{array}$ & $\begin{array}{l}\text { Epiderme do colmo da cana } \\
\qquad \mathrm{n}(\%)\end{array}$ & $\begin{array}{l}\text { Epiderme da folha da cana } \\
\qquad \mathrm{n}(\%)\end{array}$ \\
\hline Oidium sp & $2(3,03)$ & $8(3,94)$ & - & - \\
\hline Cladosporium sp & $6(9,09)$ & $27(13,30)$ & $15(16,13)$ & $16(12,13)$ \\
\hline Fusarium sp & $31(46,97)$ & $72(35,48)$ & $24(25,81)$ & $29(21,97)$ \\
\hline Geotrichum sp & $16(24,24)$ & $40(19,70)$ & - & - \\
\hline Mucor sp & $4(6,06)$ & $13(6,40)$ & - & $24(18,18)$ \\
\hline Penicillium sp & $7(10,61)$ & $23(11,33)$ & $9(9,68)$ & $12(9,09)$ \\
\hline Aspergillus sp & - & $11(5,42)$ & $32(34,41)$ & $43(32,57)$ \\
\hline Rhizopus sp & - & $9(4,43)$ & $13(13,97)$ & $8(6,06)$ \\
\hline Totais & 66 & 203 & 93 & 132 \\
\hline
\end{tabular}

Tabela 3. Freqüência de culturas positivas da conjuntiva de 100 trabalhadores* durante o corte de cana-de-açúcar, no município de Monte Belo - MG, em novembro de 2003

\begin{tabular}{|c|c|c|c|c|c|}
\hline \multirow[t]{2}{*}{ Fungos isolados } & \multicolumn{3}{|c|}{ Número de isolamentos } & \multirow{2}{*}{$\begin{array}{c}\text { Freqüência entre } \\
\text { fungos isolados ( \% ) }\end{array}$} & \multirow{2}{*}{$\begin{array}{c}\text { Freqüência entre } \\
\text { total de trabalhadores (\%) }\end{array}$} \\
\hline & Único & Associado & Total & & \\
\hline Fusarium sp & 28 & 3 & 31 & 45,59 & 31 \\
\hline Geotrichum sp & 15 & 1 & 16 & 23,53 & 16 \\
\hline Penicillium sp & 6 & 1 & 7 & 10,30 & 7 \\
\hline Cladosporium sp & 6 & 0 & 6 & 8,82 & 6 \\
\hline Mucor sp & 4 & 0 & 4 & 5,88 & 4 \\
\hline Oidium sp & 2 & 0 & 2 & 2,94 & 2 \\
\hline Candida sp & 0 & 1 & 1 & 1,47 & 1 \\
\hline Rhodotorula sp & 0 & 1 & 1 & 1,47 & 1 \\
\hline Totais & 61 & 7 & 68 & 100 & 68 \\
\hline
\end{tabular}


pécimes clínicas foram colhidas do fundo do saco conjuntival inferior de ambos os olhos.

Poucos são os trabalhos que visam o conhecimento da microbiota micótica normal do olho humano ${ }^{(6-9)}$. Com relação a trabalhadores rurais, a literatura registra número ainda menor $^{(8-9)} \mathrm{em}$ contraposição com trabalhadores de diversas áreas urbanas ${ }^{(10-13)}$.

Entre as doenças fúngicas que trazem preocupações acarretando prejuízos no setor canavieiro no sul de Minas, destacamse a ferrugem e o carvão, provocados respectivamente pelos fungos Puccinia melanocephala e Ustilago scitaminea. No presente trabalho não foram isolados nenhuma destas espécies indicando de maneira indireta, que o canavial analisado se encontra livre destes fitopatógenos ${ }^{(14)}$.

A prática dos trabalhadores do corte de cana-de-açúcar, na maioria das vezes, exercerem suas atividades sem equipamentos de proteção individual, os expõe a riscos inerentes ao trabalho uma vez que tal atividade ocorre no canavial pósqueimada e com grande quantidade de cortadores, o que possibilita a contaminação do globo ocular pela microbiota anemófila e folhas, com certa facilidade. Haja visto os freqüentes acidentes que ocorrem com pequenos fragmentos vegetais.

No presente estudo foi observado um maior número de trabalhadores do sexo masculino apresentando um ou mais gêneros de fungos no saco conjuntival. Em certos casos de ceratomicose existem fatores que predispõem pessoas do sexo masculino mais do que as do sexo feminino, em virtude de uma maior exposição daqueles a tais fatores ${ }^{(10,15)}$, entretanto, no presente estudo tais fatores poderiam ser atribuídos à forma mais agressiva de manipulação e menos cuidados protetores dos olhos em relação às mulheres. Quanto à faixa etária, a média situou-se em torno de 35,2 anos, independentemente do sexo. Neste sentido, resultados encontrados por alguns autores são semelhantes aos desta investigação onde indivíduos do sexo masculino foram mais acometidos, talvez em razão da maior atividade extra-muros do homem em nosso meio ${ }^{(7)}$.

Ainda com relação à incidência de fungos por grupo etário, pode-se observar que houve uma certa homogeneidade, em termos percentuais, de culturas positivas. Entretanto, foi constatada a existência de dois picos (31-40 e 51-60) (Tabela 1), o que discorda dos encontrados por alguns autores ${ }^{(16-17)}$. Estes dados contrastantes podem ser explicados, pelo menos em parte, pelos diferentes números de indivíduos analisados nestas faixas etárias, demasiadamente reduzido ${ }^{(7)}$.

A pesquisa de fungos anemófilos, agentes comprovadamente de patologias oculares oportunistas e ocasionais, revelou um perfil semelhante a muitos trabalhos descritos na litera$\operatorname{tura}^{(8-9)}$.

A investigação de fungos presentes no ar é de particular importância uma vez que a concentração maior ou menor de esporos, pode refletir o grau de sensibilização alérgica em indivíduos atópicos com manifestações de asma e rinite ${ }^{(17)}$. Quando ocorrem modificações ambientais em função de mudanças de temperatura, umidade e local de trabalho, a microbiota conjuntival pode carrear esporos do ar, transitoriamente, sem sofrer danos. Entretanto, quando há um desequilíbrio entre a microbiota ocular residente e a transitória, doenças podem aparecer ${ }^{(18)}$.

Na presente investigação Fusarium sp e Geotrichum sp, seguidos de Cladosporium sp e Penicillium sp, foram os fungos filamentosos de maior incidência isolados da conjuntiva ocular dos trabalhadores investigados. Coincidentemente, estes também foram os principais fungos isolados do ar. Neste aspecto, é importante ressaltar que até 1957 o gênero Fusarium era considerado um fungo saprófita. A partir de então, alguns autores demonstraram que espécies deste gênero poderiam causar patologias ${ }^{(19)}$. A partir da década de 70 têm sido relatados, com maior freqüência, infecções disseminadas por estes agentes, especialmente em pacientes imunodeprimidos ${ }^{(20)}$.

Dentre os fungos produtores de ceratomicoses o gênero Aspergillus aparece em destaque, seguido por Fusarium, Curvularia e Cândida ${ }^{(21)}$. Entretanto, em nossos achados, como anteriormente referido, Fusarium, coincidindo com o trabalho de Vieira ${ }^{(9)}$ e diferindo dos dados de Scarpi, foi o gênero de maior incidência em conjuntivas normais ${ }^{(8)}$.

É importante ressaltar que os gêneros Aspergillus e Rhizopus não foram isolados da conjuntiva normal de nenhum dos trabalhadores examinados apesar de serem encontrados na microbiota anemófila, do colmo e das folhas do canavial. Com relação ao Aspergillus, nossos dados contrastaram com o de outro autor ${ }^{(8)}$, onde este gênero obteve o segundo lugar, tanto como isolamento único quanto em associação na conjuntiva ocular. Em adição, há relato de valores substanciais deste gênero em conjuntivas de trabalhadores em ambientes canavieiros, mas números bastante reduzidos no ar, no colmo e folhas $\left(4,1\right.$ e 1 isolados, respectivamente) ${ }^{(9)}$. A explicação para tais discrepâncias podem ser explicadas pelas diferenças do meio ambiente, variações na umidade relativa do ar, época e técnica de coleta do material, entre outras ${ }^{(5,22-24)}$

Quanto ao gênero Rhizopus, nossos achados são semelhantes aos descritos na literatura, tanto para isolados de conjuntiva como do $\operatorname{ar}^{(8-9,17,25-27)}$.

\section{CONCLUSÕES}

- Das amostras colhidas do saco conjuntival de ambos os olhos de 100 trabalhadores no corte de cana-de-açúcar em Alfenas - MG, a maioria (64\%) resultou em cultivo positivo para fungo.

- Das 64 culturas positivas, 54 (84,38\%) foram de indivíduos do sexo masculino e $10(15,62 \%)$, do sexo feminino.

- Na maioria dos trabalhadores $(93,3 \%)$ detectou-se apenas um gênero de fungo, em 3 , dois gêneros e apenas 1 , três gêneros.

- Em ordem decrescente, Fusarium (43,76\%), Geotrichum (23,44\%) Cladosporium $(9,38 \%)$ e Penicillium $(7,81 \%)$, foram os fungos isolados com maior incidência na conjuntiva ocular.

- Os fungos com maior ocorrência na conjuntiva foram também, na mesma ordem, os mais isolados do ar.

- Na epiderme e colmo da cana-de-açúcar, os principais isolados foram Aspergillus, Fusarium, Mucor e Penicillium. 


\section{ABSTRACT}

Aim: To evaluate the incidence of fungi in the ocular conjunctiva of sugar-cane cutting workers as well in a sugar-cane plantation environment. Place: Monte Belo - MG, Eye Clinic, Laboratory of Physiology and Biology of Microorganisms of Unifenas, Alfenas - MG. Methods: Swabs were made from the ocular conjunctiva of one hundred workers of both sexes and material was collected from leaves, stalk, and the environmental air from the sugar-cane plantation. These were inoculated in specific mold media. After incubation at $25^{\circ} \mathrm{C}$ for a period of fifteen days, the plates were analyzed and colony forming units (UFC) were identified using conventional mycological techniques. Results: Of one hundred workers involved in this research, 64 presented one or more genera of fungi, 54 $(84.38 \%)$ being identified in males and $10(15.62 \%)$ identified in females. The separation of the workers by age range showed that the prevalence of observed fungi by age was not uniform. The highest incidences were found in advanced age ranges, the increase of positivity by age being considered statistically significant $(\mathrm{p}<0.05)$. The lowest prevalence $(50 \%)$ was found in the 11-20-year-old interval, which presents the lowest number of examined persons. The highest positivity was verified in the 61-79 year interval. In 60 workers $(93.75 \%)$ only one genus was isolated; in $3(4.69 \%)$ two genera, and in only one worker $(1.56 \%)$ three genera were isolated. The most prevalent isolated fungi were Fusarium sp (43.76\%) and Geotrichum sp (23.44\%), followed by Cladosporium sp (9.38\%), Penicillium sp $(7.81 \%)$, Mucor sp $(9.38 \%)$ and Oidium sp (7.81\%). The most common genus founded in leaves, stalk and air were Aspergillus, Penicillium, Fusarium, Cladosporium and Rhizopus. Conclusion: The environmental conditions, the socioeconomic status and the general and personal poor hygienic conditions, together with lack of information about prophylactic standards, surely led to the high incidence of ocular conjunctiva fungus isolation $(67 \%)$. The incidence was much higher in males $(84.38 \%)$ as $(15.62 \%)$ in females. The anemophilious fungi and those isolated from stalk and leaves were similar to those already described in the literature. The isolated filamentous fungi were much more numerous than yeast, there being some correlation between these and the conjunctiva isolates.

Keywords: Fungi/isolation \& purification; Conjunctiva/microbiology; Eye diseases; Saccharum; Agricultural worker's diseases

\section{REFERÊNCIAS}

1. Vaughan DG, Tabbara KF. Prevention of ocular infections. In: Tabbara KF, Hyndiuk RA. Infections of the eye. Boston: Little Brown; 1986. p.13-9.

2. Birge HL. Oculr aspects of mycotic infections. Arch Ophtalmol. 1957;47:354-82.
3. Gugnani HC, Copta S, Talwar RS. Role of opportunistic fungi in ocular infections in Nigeria. Mycopathologia. 1977;65(1-3):155-66.

4. Kolodner H. Fungal corneal ulcers. Int Ophthalmol Clin. 1984;24(2):17-25.

5. Jones DB. Strategy for initial management of suspected microbial keratitis. In: Barraquer JI, Binder PS, Bruxton JN, Fine M, Jones DB, Laibson PR, et al. Symposium on medical and surgical diseases of the cornea. St Louis: C.V. Mosby; 1980. p.86-119.

6. Azevedo ML. Investigações preliminares sobre a microflora ocular. Arq Bras Oftalmol. 1962;25(1):41-7.

7. Costa ML, Galvão PG, Lage J. Flora micótica da conjuntiva de indivíduos normais. Rev Bras Oftalmol. 1975;34(4):674-82.

8. Scarpi MJ. Microbiota fúngica da conjuntiva normal de trabalhadores no corte de cana-de-açúcar. Rev Bras Oftalmol. 1985;44(1):56-65.

9. Vieira LA, Belfort Jr RB, Fischman OF, Scarpi M. Estudo da flora fúngica da conjuntiva normal, da cana-de-açúcar e de anemófilos da região canavieira de Santa Rita - Paraíba (Brasil). Arq Bras Oftalmol. 1989;52(3):63-7.

10. Salera CM, Tanure MA, Lima WT, Campos CM, Trindade FC, Moreira JA. Perfil das ceratites fúngicas no Hospital São Geraldo Belo Horizonte - MG. Arq Bras Oftlamol. 2002;65(1):9-13.

11. Foster CS, Fungal keratitis, Infect Dis Clin North Am. 1992;6(4):851-7.

12. Ando N, Takatori K. Fungal flora of the conjunctival sac. Am J Ophthalmol. 1982;94(1):67-74.

13. Lima ALH, Nishi M, Lottemberg CL, Guiudugli T. Úlceras de córnea em serviço de referência. Arq Bras Oftalmol. 1988;51(3):118-20.

14. Tokeshi H. Doenças da cana-de-açúcar (híbridos de Saccharum spp.). In: Kimati H, Amorim L, Bergamin Filho A, Camargo LE, Rezende JA. Manual de Fitopatologia. São Paulo: Agronômica Ceres; 1997. p.207-25.

15. Kelly LD, Pavan-Langston D, Baker AS. Keratomycosis in a New England referral Center: spectrum of pathogenic organisms and predisposing factors. In: Bialasiewics AA, Schall KP, editors. Infectious diseases of the eye. Stoneham: Butterworth-Heinemann; 1994. p.184-90.

16. Williamson J, Gordon AM, Wood R, Dyer AM, Yahya A. Fungal flora of the conjunctival sac in health and disease: influence of topical and systemic steroids. Brit J Ophthalmol. 1968;52(2):127-37.

17. Mezzari A, Perin C, Santos Júnior SA, Bernd LAG, Di Gesu G. Os fungos anemófilos e sensibilização em indivíduos atópicos em Porto Alegre, RS. Rev Assoc Med Bras. 2003;49(3):270-3.

18. Gregorio CE, Feitosa VL, Candido AL, Oliveira ML. Estudo da microbiota e dos tipos celulares encontrados na secreção conjuntival de olhos clinicamente normais [dissertação]. Aracaju: Universidade Federal de Sergipe; 1992.

19. Godoy P, Colombo AL. Biologia e relevância clínica das espécies do gênero Fusarium spp. Prat Hospitalar. 2004;11(34):136-40.

20. Guarro J, Gené J. Opportunistic fusarial infections in humans. Eur J Clin Microbiol Infect Dis. 1995;14(9):741-54.

21. Calvo MCR, Rezusta A, Gil J. Infecciones oculares por el género Alternaria [texto na Internet]. Madrid: Sociedad Espanolas de Enfermedad Infecciosas y Microbiologia Clinica; 2004 [citado 2001 Jun 27]. Disponível em: http:// www.seimc.org/control/revi_Mico/alterna.htm

22. Wilson LA, Ahern DG, Jones DB, Sexton RR. Fungi from the normal outer eye. Am J Ophthalmol. 1969;67(1):52-6.

23. Mitsui Y, Hanabusa J. Corneal infections after cortisone therapy. Br J Ophthalmol. 1955;39(4):244-50.

24. Nema HV, Ahuja DP, Mohapatra LN. Mycotic flora of the conjuctiva. Am J Ophtalmol. 1966;(5):968-70.

25. Mezzari A, Perin C, Santos Junior SS, Di Gesu GM, Bernd LAG. Caracterís-

ticas dos esporos de fungos anemófilos em Porto Alegre. Rio Grande do Sul, Brazil. Rev Inst Med Trop São Paulo. 2002;44(5):269-72.

26. Trindade RC, Bonfim ACR, Resende MA. Conjunctival microbial flora of clinically normal persons who work in a hospital environment. Braz J Microbiol. 2000;31(1):12-6.

27. Schoenlei-Crusius IH, Trufem SFB, Grandi RAP, Milanez AI, Pires-Zottarelli CLA. Airbone fungi in the region of Cubatão, São Paulo State, Brazil. Braz J Microbiol. 2001;32(1):61-5. 